\title{
Estudio de calefón solar mediante el desarrollo de un dispositivo de adquisición de datos
}

Rolando L Benítez Alonso ${ }^{1}$; Noemi Sogari²; Eduardo J Ricciardi ${ }^{3}(*)$

\section{Resumen:}

en la búsqueda de tecnologías asequibles para familias rurales y con el fin de aprovechar la energía solar, dadas las condiciones climáticas de la región se construyó un calefón solar con materiales de bajo costo. Con el objetivo de estudiar su funcionamiento y mejorar su rendimiento, se diseñó un sistema de adquisición de datos utilizando un microcontrolador Arduino y distintos transductores para realizar el monitoreo del sistema y la medición de parámetros. Se midió la temperatura del agua a la entrada y la salida del sistema obteniendo un promedio de $20^{\circ} \mathrm{C}$ y $60^{\circ} \mathrm{C}$, respectivamente. $\mathrm{El}$ tanque utilizado tiene una capacidad de 80 litros y considerando un periodo de funcionamiento de 7 horas de exposición de la luz solar, se ha logrado calentar aproximadamente 40 litros de agua, llegando una temperatura final de $45^{\circ} \mathrm{C}$. Considerando los materiales utilizados, se obtuvo resultados satisfactorios

Palabras claves: calefón, energía solar, temperatura.

\section{INTRODUCCIÓN.}

En la actualidad, las fuentes de energías renovables se han convertido en alternativas asequibles para el desarrollo de aplicaciones que favorecen el cuidado del medio ambiente y la economía local. La construcción de calefones solares con materiales de bajo costo tiene como objetivo poner al alcance de las familias de escasos recursos el aprovechamiento de la energía solar, para el

\footnotetext{
(*) Grupo de Investigación, Desarrollo y Transferencia de Energías Sustentables y del Medio Ambiente (GIESMA) Facultad de Ciencias Exactas y Naturales y Agrimensura. Universidad Nacional del Nordeste (UNNE). Av. Libertad 5460 C.P. 3400 Corrientes, Argentina.

${ }^{1}$ Bo San Gerónimo. Avd. Laprida 716; rololeonel88@gmail.com.

${ }^{2}$ Bo San Martín; noemisogari@gmail.com.

${ }^{3}$ Bo 17 de agosto; ricciardi.ej@gmail.com.
} 
calentamiento de agua potable mediante la utilización de una fuente de energía inagotable y de bajo costo.

El funcionamiento del calefón solar se basa en el aprovechamiento de la energía calórica proveniente de la radiación infrarroja. Los colectores funcionan principalmente en base al espectro visible e infrarrojo del espectro solar. ${ }^{1}$

Se compone básicamente de una caja o marco con aislamiento, una cubierta de vidrio o de plástico que es la ventana por donde ingresa la radiación solar, una placa absorbedora y una red de cañerías de color negro. La radiación solar es absorbida por la placa que está construida de un material que transfiere rápidamente el calor a un fluido que circula a través de tubos. El agua fría ingresa a la red de cañerías del colector desde el tanque, por acción de la gravedad, se calienta mediante los absorvedores y reingresa al tanque con una temperatura más alta. Este proceso se lleva a cabo de manera continua y es denominado efecto termosifón.

La cubierta transparente transmite una gran cantidad de la luz de onda corta del espectro solar y al mismo tiempo, sólo deja pasar muy poca radiación de onda larga, que es el calor emitido por la superficie absorbedora, produciendo un efecto invernadero. Además, la cubierta evita que el viento pueda disipar, por convección, el calor colectado. Junto al marco of recen protección de las condiciones ambientales adversas. La caja, debidamente aislada, debe estar orientada en forma perpendicular a los rayos del sol. El agua calentada se acumula en un tanque aislado para evitar que el agua se enfríe durante la noche. ${ }^{2}$

Para el estudio del funcionamiento del calefón solar, se ha diseñado un dispositivo de adquisición de datos, que cuenta con diferentes transductores electrónicos para la medición de humedad, temperatura ambiente, humedad relativa, presión atmosférica, temperatura del agua, irradiancia y un sistema de almacenamiento y transmisión inalámbrica de datos mediante módulos de tarjeta microSD y conexión a internet, respectivamente.

\section{MATERIALES Y MÉTODOS}

La construcción del calefón se llevó a cabo con materiales de fácil adquisición en tiendas locales y otros que fueron reciclados. Se utilizó madera y chapadur para la fabricación de la caja absorbedora, la placa transparente de policarbonato y la parrilla de caños de polietileno de baja densidad (PEBD). Se aisló el interior de la caja con papel aluminio y poliestireno expandido (telgopor).

Se dispuso de un tanque acumulador de agua estanco de 80 litros de volumen, que también fue aislado con papel aluminio y poliestireno expandido (telgopor).

Se ubicó el colector en dirección norte, con un ángulo de inclinación de $37^{\circ}$ con respecto al suelo (figura 1).

E1 dispositivo de adquisición de datos se diseñó entorno a un microcontrolador en plataforma Arduino, específicamente el Arduino Mega2560. Para la medición de 
temperatura del agua, humedad relativa, temperatura ambiente, presión atmosférica, caudal de agua e irradiancia se hizo uso de diferentes transductores. El dispositivo dis-

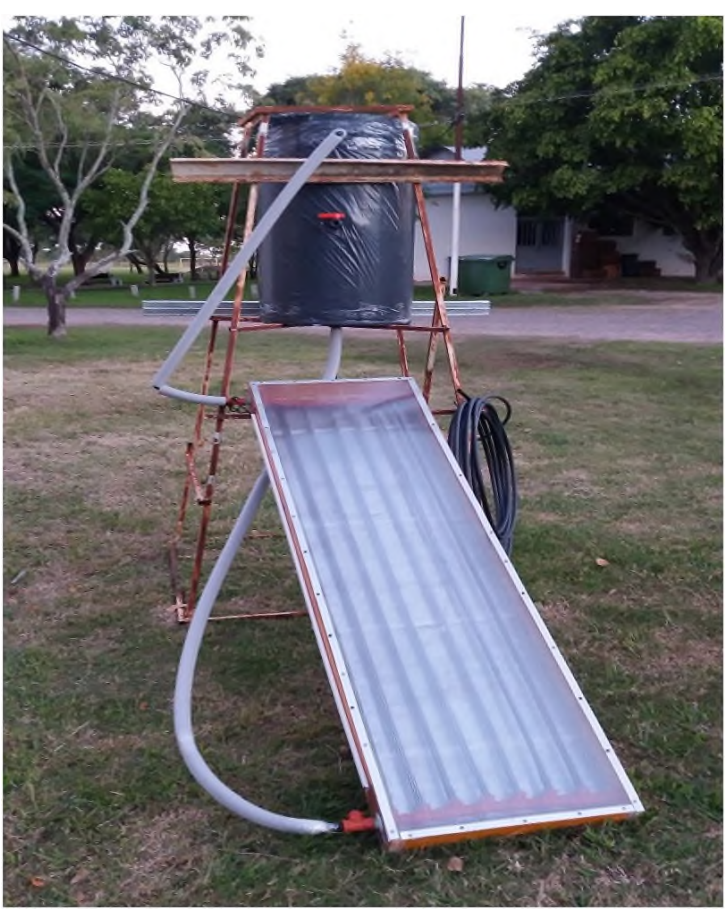

Figura 1: Colector construido.

\section{RESULTADOS Y DISCUSIÓN}

Se construyó un colector de $2 \mathrm{~m}$ de largo y $54 \mathrm{~cm}$ de ancho y un volumen aproximado de 2 litros.

La adquisición de datos se realizó en días soleados y totalmente despejados, para verificar el comportamiento del sistema en las mejores condiciones posibles. Se tomó mediciones en distintos días, en un intervalo de 7 horas de funcionamiento, entre las 10:00 hs y las 17:00 hs, franja horaria para la cual los rayos del sol inciden sobre el colector. pone de un protocolo de almacenamiento y transmisión de datos por vía wifi, lo que permite monitorear el funcionamiento del sistema (figura 2).

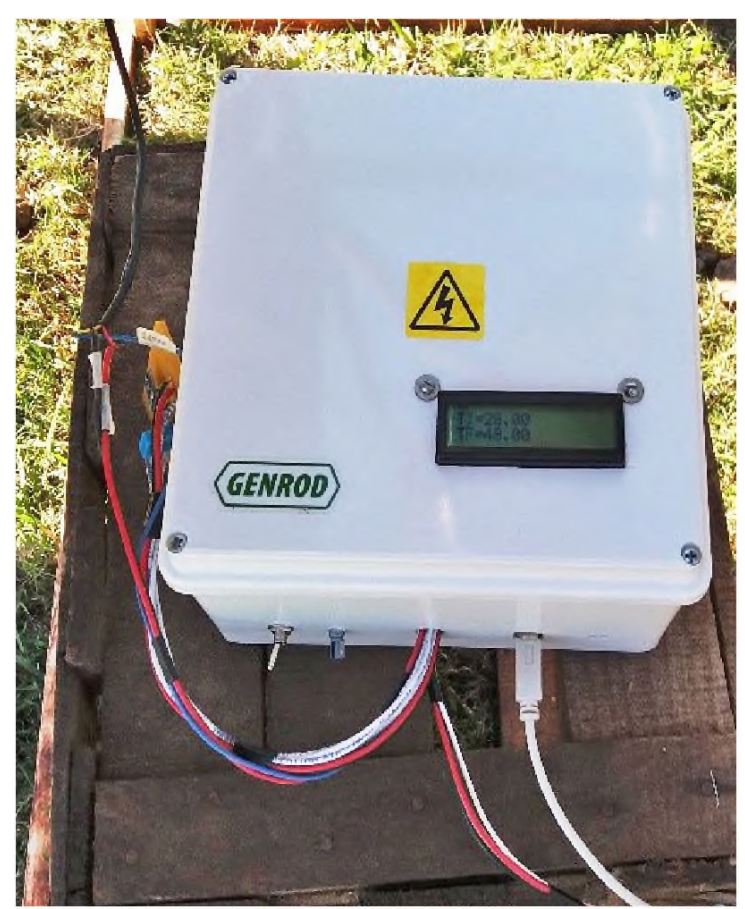

Figura 2: Dispositivo de adquisición de datos

En promedio, la temperatura del agua proveniente de la red, registró un valor de 20 ${ }^{\circ} \mathrm{C}$.

La temperatura máxima del agua registrada a la salida fue de $71.5^{\circ} \mathrm{C}$, para una temperatura ambiente variando de $21^{\circ} \mathrm{C}$ a $25^{\circ} \mathrm{C}$.

La temperatura máxima de agua alcanzada en el tanque acumulador fue de $45^{\circ} \mathrm{C}$. Se llegó a calentar aproximadamente unos 45 litros de agua, de un total de 80 litros de agua. Se debe considerar que el agua caliente que ingresa al tanque, por efecto termosifón, se mezcla con el agua fría de su interior. 


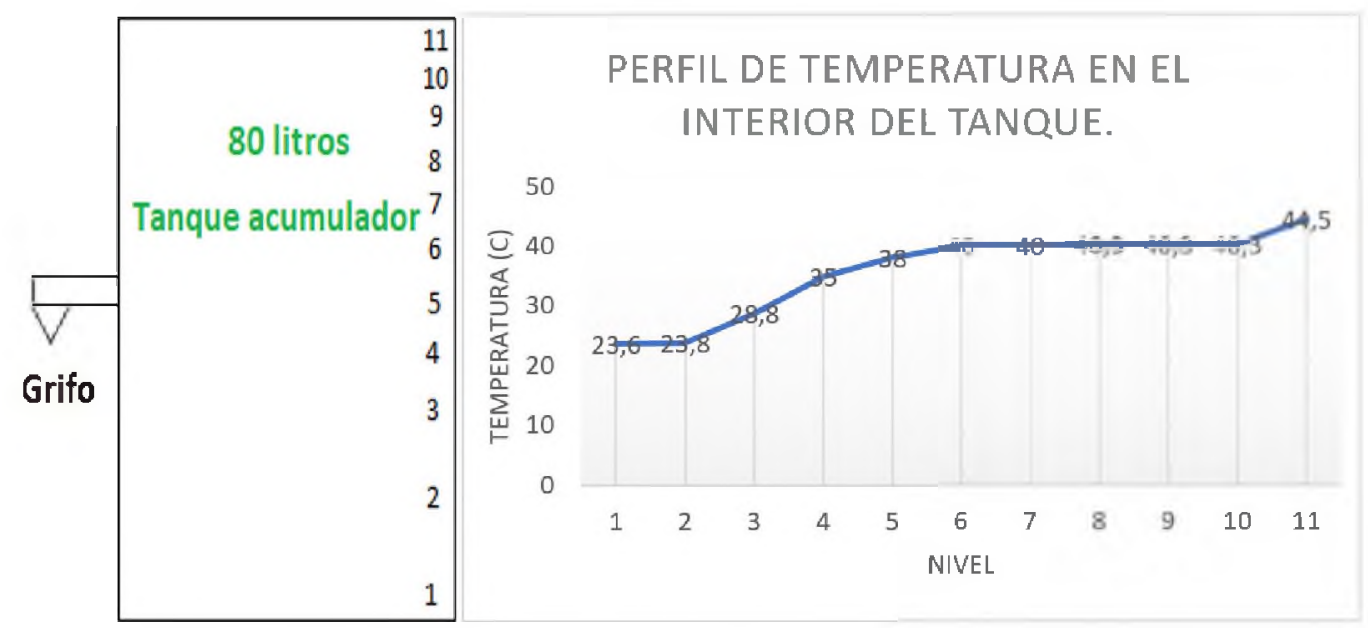

Figura 3: Medición de temperatura en el interior del tanque acumulador.

Perfil de temperatura de agua a la entrada y salida del colector.

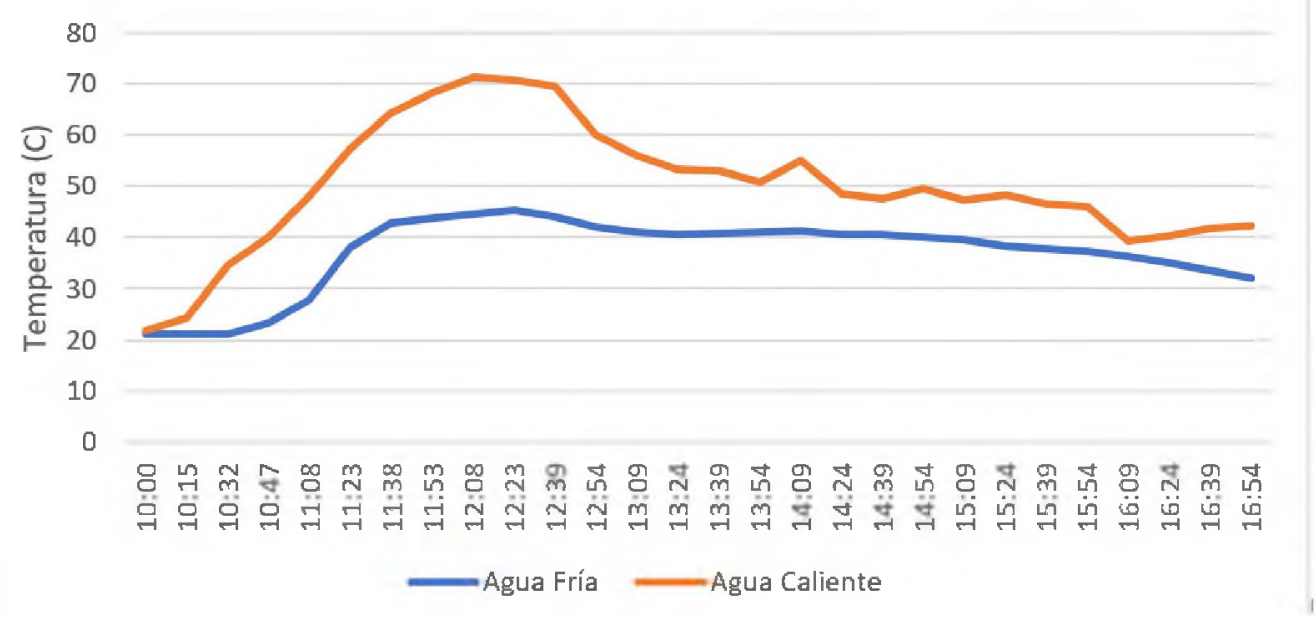

Figura 4: Curva de temperatura de agua a la entrada y salida del sistema colector.

Las curvas demuestran un funcionamiento acorde a la información teórica consultada en el material bibliográfico. Los datos presentados fueron promediados de todos los valores obtenidos durante las mediciones realizadas en días diferentes. Se tomaron mediciones de 5 días.

No se tuvo en cuenta las pérdidas de calor por convección debido a fallas en la aislación y las ocasionadas por la evaporación del agua en la cañería.

\section{CONCLUSIÓN}

Los resultados arrojados por las pruebas fueron satisfactorios, considerando los materiales utilizados y haciendo una com- 
paración con dispositivos industriales de grandes prestaciones y mejores rendimientos. Las temperaturas obtenidas podrían ser utilizadas con fines de limpieza y aseo personal, por citar algunas aplicaciones.

El dispositivo electrónico diseñado para la toma de datos pudo servir para estudiar el comportamiento del calefón solar construido.

Los materiales que fueron utilizados son de bajo costo. Se prevé construir más calefones solares, con la consigna de minimizar gastos, pero con mejores prestaciones.

\section{ABSTRACT}

In the search for affordable technologies for rural families and in order to take advantage of solar energy, given the climatic conditions of the region, a solar hea- ter was built with low-cost materials. In order to study its operation and improve its performance, a data acquisition system was designed using an Arduino microcontroller and different transducers to monitor the system and measure parameters. The water temperature at the inlet and outlet of the system was measured obtaining an average of $20^{\circ} \mathrm{C}$ and $60^{\circ} \mathrm{C}$, respectively. The tank used has a capacity of 80 liters and considering a period of operation of 7 hours of exposure to sunlight, it has been possible to beat approximately 40 liters of water, reaching a final temperature of $45^{\circ} \mathrm{C}$. Considering the materials used, satisfactory results were obtained.

Keywords: water heater, solar energy, temperature. 


\section{BIBLIOGRAFÍA}

1- Placco, C.; Saravia, L.; Cadena, C. En: Colectores solares para agua caliente. Instituto de Investigaciones en Energía No Convencional (INENCO). Universidad Nacional de Salta (UNSa). Consejo Nacional de Investigaciones Científicas y Técnicas (CONICET). Salta. Año 2008.

2- Duffie, J. A.; Beckman, W. A.; Solar Engineering of Thermal Processes; 4ta edición; John Wiley \& Sons, Inc., Hoboken, New Jersey (EEUU); ISBN 978-1-118-43348-5; pp. 479-499; 2013.

3- San Juan G. (2008). Calentador de Agua Solar. Manual del Usuario. Tecnología sencilla. Facultad de Arquitectura y Urbanismo. Universidad Nacional de la Plata. ISBN: 978-950-34-0484-3
4- De la Horra Killmer, M. (2013). Proyecto de Fin de Carrera: Sistema de Adquisición de Datos basado en la plataforma Arduino. Aplicaciones a Matlab, Simulink y Android. Universidad Carlos III de Madrid. Leganés - España.

5- Fernández Díez P. (2003). Procesos Termosolares en baja, media y alta temperatura. Departamento de Ingeniería Eléctrica y Energética. Universidad de Cantabria. Santander - España.

6- Manual de Construcción, Calentador de Agua Solar. Unión de Esfuerzos para el Campo, AC. (UDEC). Tequisquiapan, Qro, México. 